\section{P63 TREATMENT OF PELVIC INFLAMMATORY DISEASE} USING SHORT-COURSE AZITHROMYCIN

doi:10.1136/sextrans-2012-050601c.63

${ }^{1} \mathrm{~L}$ Cunningham, ${ }^{*}{ }^{1} \mathrm{~A}$ Murray, ${ }^{2} \mathrm{P} \mathrm{J}$ Horner. ${ }^{1}$ University Hospitals Bristol NHS Foundation Trust, Bristol, UK; ${ }^{2}$ University Hospitals Bristol NHS Foundation Trust/University of Bristol, Bristol, UK

Background In our centre, a novel 5-day regimen of azithromycin (1 g stat then $500 \mathrm{mg}$ daily for 4 days with or without metronidazole and/or ceftriaxone) is used and has been approved regionally. This is administered as an alternative regimen in selected cases of pelvic inflammatory disease (PID), usually those with milder symptoms and signs.

Aims and Objectives We assessed the use and efficacy of short course azithromycin in PID. We also assessed follow-up rates and adherence for all cases of PID.

Methods Retrospective case note review of 129 female patients coded as PID during the period 1 July to 31 December 2011. Data were collected and transferred to Excel for analysis.

Results 109/129 (84.5\%) of women were diagnosed with PID based on symptoms and signs as described in the BASHH PID guideline. Chlamydia was subsequently diagnosed in 10/108 (9.3\%) and gonorrhoea in 5/108 (4.6\%). Chlamydia antibody titres of $>1$ in 256 were found in $20 / 65$ (30.8\%) of patients. Taking into account missing data, 39/51 (76.5\%) patients completed the antibiotic course and 64/ $78(82 \%)$ reported full or partial resolution of symptoms. 14/85 $(16.5 \%)$ required further PID treatment, eight of whom had sexual intercourse with an untreated partner (see abstract P63 table 1).

Discussion In this study, 32/109 (29.4\%) women were treated for PID with azithromycin either in combination or alone. Overall, the majority of women completed treatment and reported a full or partial improvement in symptoms. Follow-up rates were good but documentation was inadequate. Comparisons between regimens are limited due to missing data. Further work will look at novel follow-up methods such as routine telephone contact as well as standardisation of follow-up documentation.

Abstract P63 Table 1 Antibiotic regime for PID treatment and response

\begin{tabular}{lllllll}
\hline $\begin{array}{l}\text { Principle } \\
\text { antimicrobial }\end{array}$ & $\begin{array}{l}\text { Treated } \\
(\%)\end{array}$ & $\begin{array}{l}\text { Follow-up } \\
\text { attended } \\
(\%)\end{array}$ & $\begin{array}{l}\text { Completed } \\
\text { course }\end{array}$ & & $\begin{array}{l}\text { Full/partial } \\
\text { clinical } \\
\text { response }\end{array}$ & \\
\hline & & & & $\begin{array}{l}\text { Missing } \\
\text { data }\end{array}$ & $\begin{array}{l}\text { Missing } \\
\text { data }\end{array}$ \\
Azithromycin & $32(29.4)$ & 21 & $5 / 8$ & 13 & $14 / 19$ & 2 \\
Ofloxacin & $71(65.1)$ & 59 & $32 / 40$ & 19 & $46 / 55$ & 4 \\
Doxycycline & $2(1.8)$ & 2 & $0 / 2$ & 1 & 2 & 0 \\
Other & $4(3.7)$ & 3 & 2 & 1 & 2 & 1 \\
Total & 109 & $85(78)$ & 39 & 34 & 64 & 7 \\
\hline
\end{tabular}

P64 HIGH RATES OF RECTAL CHLAMYDIA TRACHOMATIS IN WOMEN REPORTING ANAL SEX IN THE GUM CLINIC

doi:10.1136/sextrans-2012-050601c.64

S Simon, ${ }^{*}$ M Hassan-Ibrahim, S Soni. Brighton and Sussex University Hospitals NHS Trust, Brighton, UK

Background Nucleic acid amplification (NAAT) testing for Neisseria gonorrhoeae (GC) and Chlamydia trachomatis (CT) is now widely available but not licensed for use at non-genital sites. Despite this, high rates of pharyngeal and rectal GC and CT have been demonstrated in MSM. Currently there is little data on the prevalence of infection at these sites in women reporting oral and anal sex.

Aim To determine the prevalence of pharyngeal and rectal CT and GC in women attending GUM.
Methods Between 1 June and 31 October 2011 all women reporting oral and anal sex were offered pharyngeal and blind rectal swabs as well as cervical testing for CT and GC. Samples were analysed using the BD ProbeTecTM CT/GC Ox Amplified DNA assay in the BD ViperTM system. All women with positive GC NAAT results had swabs sent for GC culture and sensitivities.

Results Median age was 25 years (IOR 21-31) and most women were of white ethnicity. 2235 swabs were taken, 124 were excluded due to incomplete labelling. 2111 women had non-genital sampling: 165 rectal swabs only, 1652 pharyngeal swabs only, 147 both sites. In nine and five women the pharynx and rectum were the sole sites of CT infection respectively. In two women, the pharynx was the sole site of GC infection. All women were asymptomatic at throat and rectum (see abstract P64 table 1).

Discussion Rectal CT rates were high among women reporting anal sex. As the antibiotic treatment of cervical and rectal CT differs, this pool of infection could remain untreated. We recommend routinely asking about anal sex and testing where appropriate. Rates of pharyngeal CT were low but higher than GC overall. Although 11 women had pharyngeal CT/GC only, and would have been missed, the cost of detecting one of these infections was $£ 1727$ and this has precluded ongoing screening.

Abstract P64 Table 1 CT and GC prevalence at different sites

\begin{tabular}{lccc}
\hline & Pharynx & Rectum & \multicolumn{1}{l}{ Cervix } \\
\hline CT NAAT & $1.3 \%(23 / 1799)$ & $7.1 \%(22 / 312)$ & $6.1 \%(194 / 3073)$ \\
GC NAAT & $0.28 \%(5 / 1799)$ & $0.32 \%(1 / 312)$ & $0.49 \%(15 / 3073)$ \\
GC Culture & $0.17 \%(3 / 1799)$ & $0.32 \%(1 / 312)$ & $0.36 \%(11 / 3073)$ \\
\hline
\end{tabular}

\section{P65 SEROLOGICAL RESPONSE BETWEEN HIV-POSITIVE AND NEGATIVE COHORTS TREATED FOR EARLY SYPHILIS}

doi:10.1136/sextrans-2012-050601c.65

R Dhairyawan, ${ }^{*}$ A Almeida, M Gunathilake, B Goh. Barts and the London NHS Trust, London, UK

Introduction Successful treatment response for early syphilis involves resolution of signs and a fourfold decrease in RPR/VDRL or sero-reversion between 3 and 6 months. However, in the HIVpositive population the serological response can be unusual.

Aims To compare serological response after treatment of early syphilis between HIV-positive and negative cohorts.

Methods Retrospective cohort study of 100 HIV positive (HP) and 92 negative (HN) patients with early syphilis at an inner city GUM clinic from 2002 to 2011. Data were collected on demographics, stage of syphilis, treatment and serological response.

Results Both HP and HN cohorts were mainly MSM (72 HN, 96 $\mathrm{HP}$ ) and Caucasian (HN 76, HP 78). Median age 33 (range 20-63 years) in the HN and 37 (range 19-59 years) in the HP. Stage of syphilis: HN group, 22/92 primary, 26 secondary and 44 early latent (EL). HP group: 26/100 primary, 34 secondary and $39 \mathrm{EL}$. The $\mathrm{HN}$ group were treated with the standard regimen for their stage of disease. 49/100 of the HP group received treatment for neurosyphilis. 25/92 HN and 14/100 HP were lost to follow-up following treatment. Excluding these patients, by 6 months, 18/20 (90\%) of the HP group with primary syphilis sero-reverted or had a fourfold decrease in RPR/VDRL compared to $17 / 17$ (100\%) of the HN. By 6 months, 51/55 (93\%) of the HP group with secondary/EL syphilis had sero-reverted or had a fourfold decrease in RPR/VDRL compared to $41 / 45$ (91\%) of the HN. Median CD4 count in the HP group was 380 cells/ $\mu$ prior to treatment and 518 after treatment. There were 4 reinfections in the HN group and 10 in the HP.

Conclusions There were high rates of serological treatment success for early syphilis in both groups. However, the HP group were less 
likely to have a successful response by 6 months in primary syphilis. This study also demonstrates the need for strategies to address the large number of patients lost to follow-up.

\section{P66 TESTING FOR TRICHOMONAS VAGINALIS (TV) BY TRANSCRIPTION MEDIATED AMPLIFICATION (TMA). AN EVALUATION IN A LARGE CITY CLINIC}

doi:10.1136/sextrans-2012-050601c.66

M Lawton,* G Schembri, M Kingston. Manchester Royal Infirmary, Manchester, UK

Background Trichomonas is worldwide, the commonest curable STI. While it's prevalence in the UK is less than other areas of the world, it remains a common cause of vaginal symptoms in women. Although not routinely tested in men it can be a cause of discharge and dysuria. It may be asymptomatic in both sexes. There is debate about the significance of TV infection, over and above it's symptomatology. The majority of centres use wet-film microscopy and/or culture for the detection of TV. The sensitivity of wet-film is recognised to be low. Culture has been considered the gold standard for TV detection, but is slow and costly.

Methods Symptomatic female patients and men with recurrent/ persistent NSU were tested for TV using the same sample as the Chlamydia/gonorrhoea specimen. They were analysed using the Gen-Probe APTIMA TV assay in addition to the usual Chlamydia/ gonorrhoea AC2 assay. Patients symptomatic of discharge had wetfilm microscopy done as per standard clinical practice. Demographic data and symptomatology were recorded. Comparison was made between results from TMA and wet-film.

Results 1457 patients were tested for TV using TMA. Almost all (97\%) were women. The overall prevalence for Trichomonas, Chlamydia and gonorrhoea via TMA was 3\%, $8 \%, 1 \%$ respectively. TMA identified significantly more cases of TV compared to wet-film (41 vs 20, $p=0.009$ ). The prevalence of TV was significantly higher than gonorrhoea $(p=0.002)$. Subset analysis will be done prior to presentation.

Conclusion Testing for TV via TMA identified significantly more infections compared with the current method of detection. It's overall prevalence was much higher than gonorrhoea which is routinely screened for in asymptomatic patients. Given the same sample is used for analysis, it does not add any additional clinic time or discomfort to the patient. Cost effectiveness of using TV TMA, particularly in asymptomatic patients has not yet been evaluated.

\section{P67 ESTIMATION OF POPULATION COVERAGE OF CHLAMYDIA TESTING AMONG YOUNG ADULTS IN ENGLAND IN 2010}

doi:10.1136/sextrans-2012-050601c.67

${ }^{1} \mathrm{~A}$ Nardone, ${ }^{*}{ }^{2} \mathrm{C}$ Robinson, ${ }^{2} \mathrm{R}$ Craig, ${ }^{3} \mathrm{~S}$ Woodhall, ${ }^{3} \mathrm{~A}$ Talebi, ${ }^{4} \mathrm{C}$ Mercer, ${ }^{4} \mathrm{~A}$ M Johnson. ${ }^{1}$ Health Protection Agency; ${ }^{2}$ National Centre for Social Research; ${ }^{3}$ National Chlamydia Screening Programme, Health Protection Agency, London, UK; ${ }^{4}$ Centre for Sexual Health \& HIV Research, University College London, London, UK

Background The National Chlamydia Screening Programme (NCSP), established nationally in England in 2008, aims to prevent and control chlamydia infection in young adults under 25 years of age through opportunistic community based testing.

Aim We wished to validate the NCSP estimates of chlamydia screening coverage in the target population of young adults by comparing to self-reported chlamydia testing among participants to the Health Survey for England (HSE).
Methods Chlamydia screening coverage is calculated centrally by combining the number of tests reported from three sources of data: NCSP; sexual health clinics; and laboratories. All three sources provide data by age and sex. HSE is an annual general health survey of a nationally representative selection of households in England. In 2010 , questions on previous history of testing for chlamydia were asked of all 4259 individuals aged 16-54 who were interviewed. Analyses presented here are limited to the 725 young adults aged 16-24 years old.

Results In 2010, NCSPestimated that 2.2 million chlamydia tests were performed in England among young adults aged 15-24 years old, representing up to $33 \%$ of the target population ( $43 \%$ of females and $24 \%$ of males). In HSE $2010,44 \%$ of females (177/402) and $27 \%$ of males (87/323) reported having ever had a chlamydia test. The proportion who reported having had chlamydia test in the last year was lower for both females (27\%; 109/402) and males (17\%; 55/323).

Conclusion We have demonstrated the progress made by NCSP in achieving high national levels of coverage. Estimated coverage rates in 2010 reported by NCSP were slightly higher than those recorded by HSE which may be in part due to the inclusion of those who have had repeat chlamydia tests. The data collected by HSE has proved a valuable source of data with which to monitor the progress of NCSP in achieving national targets for testing coverage and improve the delivery of the programme.

\section{P68 IMPROVING THE MANAGEMENT OF CHLAMYDIA IN NON-GUM SETTINGS: IT TO THE RESCUE!}

doi:10.1136/sextrans-2012-050601c.68

C N C Zhou,* J Roberts, J Davies, M Longbone, G Dean. Brighton and Sussex University Hospitals, Brighton, UK

Background BASHH guidelines recommend all patients diagnosed with Chlamydia trachomatis (CT) should be given a detailed explanation of the condition, managed with appropriate antibiotics and have effective partner notification (PN). Patients tested in nonGUM settings may receive sub-optimal management. Following a 2008 audit highlighting several untreated CT cases in the Gynaecology Department (GD) we introduced a bespoke IT lab-link allowing daily downloads of results to GUM health advisers (HA). Objectives To re-audit the management of females tested for CT in GD.

Methods We performed a retrospective database analysis of all CT tests requested by any of the 11 Gynaecology consultants from June 11 to January 12. Demographic and clinical details were extracted from a prospectively collected lab. database and clinic records. Results were compared with the 2008 audit. Fisher's exact test was used to compare differences between proportions.

Results 889 tests $\{864(97.2 \%)$ negative, $16(1.8 \%)$ positive, $9(1 \%)$ not tested-incorrect swab\} were requested by GD over 29 weeks. HAs were notified of $100 \%$ of results in real time. Median time from notification of positive results to patient contact was 1 day (range 1-60). Median time from positive result to treatment was 7 days (range 1-70). This compares to an upper limit of 168 days in the 2008 audit. Recommended antibiotics were used in all cases. PN outcomes improved from $31 \%$ to $75 \%(\mathrm{p}=0.02)$ and untreated cases decreased from $38 \%$ to $18.7 \%(p=0.2)$ (see abstract P68 table 1 ).

Discussion Since the introduction of a referral pathway and automated IT lab-link, management of CT positive patients from GD has improved, in particular PN and proportion left untreated. GUM departments should have a clear pathway for the management of patients diagnosed with STIs in non-GUM settings. 